\title{
ÉTICA DE LA TIERRA Y JUSTICIA AMBIENTAL: REFLEXIONES EN TORNO A LA RESPONSABILIDAD DEL SER HUMANO EN EL DEVENIR ACTUAL, DESDE UN ENFOQUE SOCIAL Y FILOSÓFICO
}

\author{
LAND ETHIC AND ENVIRONMENTAL JUSTICE: REFLECTIONS ABOUT \\ HUMAN BEINGS' RESPONSIBILITY IN CURRENT DEVELOPMENTS \\ FROM A SOCIAL AND PHILOSOPHICAL APPROACH
}

\section{Claudia VALENZUEla SANHUEZA*}

\section{RESUMEN}

Desde hace algunas décadas se ha expuesto el deterioro de los ecosistemas y el mundo biótico en general, haciendo hincapié en el aspecto científico-técnico de la temática; sin embargo, el análisis carece de un sustrato filosófico que permita tener una visión holística acerca de la incapacidad del ser humano para contrarrestar los efectos nefastos de la industrialización y la urbanización a gran escala. Esta investigación tiene como propósito aproximarse a la discusión a través de la revisión del trabajo pionero de Aldo Leopold, denominado "Una ética de la tierra", donde en un giro dialéctico inédito en la época, el ser humano pierde su rol central de dominador de la naturaleza para ser un miembro más de ella. A partir de esta responsabilidad humana intrínseca, pensadores como Attfield, Jonas, Rawls y Habermas, reflexionan sobre los acuerdos comunes de bienestar social, donde la justicia social y ambiental es el centro del debate, considerando el bienestar actual y futuro de la sociedad y de la comunidad biótica.

Palabras clave: Ética, justicia social, justicia ambiental, acción comunicativa.

\section{ABSTRACT}

For some decades, the deterioration of ecosystems and the biotic world in general has been exposed, emphasising the scientific-technical aspect of the subject. However, the analysis lacks a philosophical ground that allows a holistic view on human's failure to counteract the harmful effects of industrialization and large-scale urbanisation. This research aims to approach the discussion through the review of Aldo Leopold's pio-

\footnotetext{
* Socióloga, Magíster en Análisis Geográfico, Universidad de Concepción, Candidata a Doctora en Geografía, Pontificia Universidad Católica de Chile. Santiago, Chile. Correo electrónico: cdvalenzuela2@uc.cl
} 
neering work called Land Ethic, where in a completely new dialectic twist at the time, human beings lose their central role as rulers of nature to become just one more of its members. From this intrinsic human responsibility, thinkers such as Attfield, Jonas, Rawls, and Habermas, reflect on common social welfare agreements, where social and environmental justice are the centre of the debate, considering the current and future wellbeing of society and the biotic community.

Keywords: Ethic, social justice, environmental justice, communicative action.

Recibido: 06.03.17. Aceptado: 09.06.17.

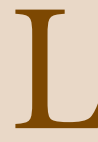

A EVIDENCIA CIENTÍFICA expone los catastróficos resultados de la ocupación intensiva de los recursos naturales, así como la presión sobre los espacios urbanos, los que se resumen en contaminación ambiental, segregación socioespacial, crecimiento incontrolado de la mancha urbana, entre muchos otros efectos. Sin embargo, estas no son problemáticas de corta data. Ya en 1949, el ecólogo y ambientalista Aldo Leopold escribía su ensayo más destacado, "La ética de la tierra", donde propone un nuevo tipo de relación entre la sociedad contemporánea y la naturaleza, extendiendo la consideración ética hacia los suelos, las aguas, las plantas, los animales o colectivamente la tierra, premisa que configura el giro ético que cambia el papel del ser humano desde conquistador de la comunidad de la tierra al de un miembro más de ella (Leopold, 2007, p. 30). Para algunos autores esta consideración cobra relevancia en este siglo, al abordar la relación establecida por la sociedad industrial globalizada con el mundo natural, y que es la principal causa de la crisis ambiental actual. Asimismo, Leopold integra la dimensión económica con un trasfondo ecológico, relación que, al incorporarse a la educación, la toma de decisiones y las políticas de desarrollo, ofrece una alternativa ecológicamente informada para sobrellevar algunos de los problemas ambientales más críticos (Rozzi, 2007).

Sin embargo, Leopold enfatiza que un sistema de conservación basado en un puro interés económico individual es desequilibrado, ya que se ignora el beneficio intangible de procesos y especies, además de la excesiva responsabilidad de los gobiernos sobre funciones que debieran ser repartidas con el sector económico y personas naturales (Leopold, 2007, p. 35). Adicionalmente, uno de los principales obstáculos para el desarrollo de una ética de la tierra se vincula precisamente con la disposición del análisis en un aspecto meramente económico, soslayando lo ética y estéticamente correcto, es decir, cuando tiende a preservar la integridad, la estabilidad y la belleza de la comunidad biótica. Esta primera aproximación fue el impulso 
inicial de una multiplicidad de trabajos inspirados en su obra, la cual se fundamentaba en tres ideas: la Tierra es una comunidad de entidades vivas, ha sido hecha para amarla y respetarla, y entrega una cosecha de cultura. Cabe destacar que las repercusiones se manifiestan hasta la actualidad, creando una nutrida literatura, y convirtiéndola en un tópico capital de las preocupaciones filosóficas actuales (Villarroel, 2007, p. 57).

Villarroel (2007) plantea que la ética ambiental surge a mediados del siglo XX incitada por la necesidad de enfrentar a dos importantes desafíos planteados por el antropocentrismo tradicional: en primer término, la presunción de superioridad moral de los seres humanos respecto de los miembros de otras especies, y en un segundo término, a la elaboración de argumentos racionales que asignaran valor intrínseco al ambiente natural y sus componentes no humanos, particularmente en el contexto de la modernidad, mediada por la técnica. Entre los trabajos pioneros que expresaron su posición frente a la crisis medioambiental emergente, se puede señalar el libro recopilatorio Silent Spring, publicado en 1962 por Rachel Carson, que retrataba la forma en que ciertos pesticidas agrícolas se infiltraban en la cadena alimentaria, afectando al medioambiente y a la salud de los seres humanos. Posteriormente, Paul Ehrlich publica en 1968 The Population Bomb, donde expone que el crecimiento incontrolado de la población humana amenaza la viabilidad de los sistemas de apoyo vital del planeta. Estos trabajos se plasmaron en la investigación liderada por Dennis Meadows en el Instituto Tecnológico de Massachusetts, que dio lugar en 1972 a The Limits to Growth, informe que recogía las preocupaciones surgidas durante la década anterior y respondía de este modo a la nueva sensibilidad medioambiental.

Paralelamente, en los albores de los años setenta se produce lo que se conoce como la "revolución de las éticas aplicadas", en que al giro lingüístico y al pragmático de la filosofía, se incorporaría el giro aplicado de la ética a las distintas actividades sociales. De este modo se fundaron la bioética, la ética económica y empresarial, la ética del desarrollo y, más tarde, la ética de los medios de comunicación, la ética de las profesiones, la infoética y un sinnúmero de reflexiones de filosofía moral, abocadas a descubrir los elementos éticos implicados en actividades como la sanitaria, la empresarial, la económica, los medios o las distintas profesiones (Cortina, 2011, p. 9).

En el contexto de la ética medioambiental, Lecaros (2013) asevera que ésta es una rama de la ética aplicada, que reflexiona sobre los fundamentos de los deberes y responsabilidades del ser humano con la naturaleza, los seres vivos y las generaciones futuras. Durante los años setenta en países 
anglosajones se comienza a utilizar la expresión Environmental Ethics para referirse a esta nueva área de la ética aplicada. Esta expresión se consolidó a partir de la conferencia titulada "Filosofía y crisis medioambiental", en la que se hacía eco de la preocupación de los primeros movimientos ecologistas de los años sesenta y del debate político-jurídico internacional. A partir de entonces el concepto de ética ambiental comenzó a conocerse como "un nuevo campo de la ética filosófica preocupada por la descripción de los valores que posee el mundo natural no humano y por la prescripción de una respuesta ética apropiada para asegurar la preservación o restauración de estos valores" (Lecaros, 2013, p. 178). El autor menciona a dos filósofos que abordaron esta dimensión de la ética y su fundamentación, Hans Jonas y Karl Apel, quienes utilizaron el término "ética de la responsabilidad". El debate filosófico en esta disciplina se ha centrado en tres grandes líneas: la primera de ellas intenta diagnosticar las causas de la crisis socioecológica en un nivel planetario, desde una perspectiva holística; la segunda línea temática incorpora los intereses morales de los seres humanos futuros o generaciones futuras, y finalmente la teoría del valor sobre la cual se sustentan las obligaciones ético-ecológicas, dividida en valores instrumentales, extrínsecos a la naturaleza y basados en los intereses humanos que fundamentan los antropocentrismos éticos y en valores intrínsecos a la naturaleza o a los seres vivos que la integran, fundamento de los biocentrismos y ecocentrismos en ética medioambiental (Lecaros, 2013).

De modo general, se estima que el pensamiento ecologista se nutre de la tensión entre la crítica a la técnica y la preocupación por conservar una naturaleza "original", siendo la articulación de estas dos corrientes la riqueza de la corriente ecologista. Siguiendo al pensamiento jonasiano, la técnica moderna ha introducido acciones de tal magnitud, con objetos y consecuencias inéditos, que el paradigma ético existente hasta ahora no resulta suficiente para abarcarlos:

Es el creciente poder humano y la progresiva comprensión de los efectos sobre este poder sobre un medioambiente limitado, es decir, la colisión que ha sobrevenido entre lo casi infinito del poder y apetecer humanos, por una parte, y lo finito de una naturaleza, por la otra, que está ahí para suministrar lo necesario, lo que ha inaugurado esta nueva dimensión de la ética (Jonas, 2001, p. 60).

$\mathrm{Al}$ respecto, se ha establecido que, en el contexto de esfera moral, comienzan progresivamente a adquirir protagonismo y relevancia tanto la acción colectiva como el sujeto colectivo, la responsabilidad por las ge- 
neraciones futuras, la precariedad de la naturaleza, es decir, asuntos que plantean nuevos cuestionamientos a las teorías éticas tradicionales (Arcas, 2007). En este sentido, Marcos (2000) señala que las cuestiones de ética ambiental están poniendo en tela de juicio a las tradiciones de pensamiento éticas más consolidadas, como es el caso de la tradición contractualista moderna, con connotados exponentes como John Rawls, quien sostiene que una sociedad es justa si sus normas de convivencia pueden ser pensadas como un contrato aceptado libremente en condiciones de igualdad para todos los afectados. Sin embargo, esto plantea una dificultad al hablar de justicia entre generaciones muy distantes o entre personas y animales no humanos, de manera que los problemas ambientales no son sólo un campo para la aplicación de normas éticas preexistentes, sino más bien una fuente de sugerencia para un nuevo pensamiento ético (Marcos, 2000).

Distanciándose de los antiguos imperativos éticos, entre los cuales el imperativo kantiano constituye el parámetro ejemplar: "Actúa de tal modo que el principio de tu acción se transforme en una ley universal", Jonas propone un nuevo imperativo: "Actúa de tal modo que los efectos de tu acción sean compatibles con la permanencia de una vida humana auténtica". La vulnerabilidad de la naturaleza sometida a la intervención tecnológica del hombre muestra una situación inusitada, pues la biósfera del planeta está expuesta a posibles alteraciones, lo cual hace imprescindible considerar que no sólo debe anhelarse el bien común, sino también el de toda la naturaleza extrahumana (De Siqueira, 2001, p. 279).

De Siqueira (2001) admite que Jonas se apartó de la concepción de la ciencia como elemento que prescinde de valores, donde el saber moderno, de fuerte predominio técnico, se acompaña de un poder inconmensurable de transformación, careciendo de una reflexión ética que equilibre el poder de la tecnociencia. Los científicos, siendo humanos, por lo general no admiten sus errores y limitaciones, lo que hace que la adopción de un nuevo paradigma sea un proceso de progresiva conversión que no implica la fuerza, sino el convencimiento lento y gradual, particularmente si la ciencia tiene una comprensión cada vez más detallada de la naturaleza. No obstante, y considerando la responsabilidad primaria del ser humano sobre los designios de la naturaleza, en la actualidad la humanidad se enfrenta a una explotación planetaria que, según Jonas, ya no cabe interpretar en la estrecha dialéctica amo-esclavo. Es así que para el autor no es sólo un dominio ejercido por los dueños de los medios de producción, sino también una sobreexplotación de la Tierra en la que participan las fuerzas del trabajo que han ido elevando su poder adquisitivo y de consumo, siendo 
los seres humanos responsables de este sistema de dominio y explotación planetaria. Esto se ve facilitado además por una automatización de la vida que ha permitido multiplicar la fuerza de trabajo del cuerpo humano para usufructuar de la Tierra a un ritmo nunca imaginado antes de la revolución industrial (González, 2015, p. 5).

En este sentido, la elección de modelos de desarrollo escasamente ha contemplado un equilibrio entre los bienes internos que se pretenden alcanzar con un desarrollo a escala humana, parafraseando a Max-Neef, y una ética que sea efectiva en el cuidado y conservación de los recursos naturales. Una discusión vinculada es la ofrecida por Sen al presentar dos concepciones del desarrollo, a las que denomina respectivamente BLAST ("blood, sweat and tears") y GALA ("getting by, with a Little assistance") (Sen, 1998). BLAST concibe el desarrollo como un proceso necesariamente cruel, mientras que para GALA es un proceso de cooperación, pues quienes actúan en el mercado son interdependientes y además los servicios públicos pueden fomentar la cooperación entre los individuos. BLAST preconiza que es necesario realizar determinados sacrificios, en términos de prestaciones sociales, de desigualdad o de autoritarismo, para lograr altos niveles de acumulación que permitan la formación de capital, donde se debe sacrificar la calidad de vida de una de parte de la población presente, e incluso de una parte de la población del futuro inmediato, para favorecer a las generaciones futuras. A juicio de Sen, BLAST presenta dos ideas base: es necesario tener en cuenta las compensaciones intertemporales y la acumulación del capital. Pero también presenta dos defectos esenciales; su desinterés por la calidad de vida del presente y del futuro inmediato, y su falta de creatividad para superar las decisiones difíciles, es decir, para inventar fórmulas que impidan tener que elegir entre el aumento de la calidad de vida de las generaciones presentes y el aumento de la productividad, al mostrar que la calidad de vida de las generaciones presentes puede ser también un factor de productividad. Por eso GALA detecta la interdependencia entre calidad de vida y productividad económica; el desarrollo social puede fomentar un crecimiento integral si se complementa con políticas favorables, es decir, el capital humano es indispensable para generar capital físico (Cortina, 2011, p. 20).

Se destaca que, en estas dos perspectivas de desarrollo, no se hace mención deliberada acerca de la inclusión de una ética de la tierra, aun cuando sí se menciona la supervivencia de las futuras generaciones. A juicio de algunos autores, y atendiendo el principio de responsabilidad jonasiano, así como el consecuencialismo de Attfield (2005), la responsabilidad del ser humano en el reino animal recae exclusivamente sobre sí mismo, teniendo 
la capacidad de cambiar el curso de la historia de la vida con sus intervenciones. A juicio de De Siqueira (2001), en una ruta que se bifurca es el caminante quien tiene la opción de elegir; un camino puede terminar en un precipicio, mientras el otro en fuentes de aguas puras, siendo estos puntos de bifurcación la esencia de la elección que presenta contornos apropiados a través de una decisión ética. El producto final obtenido depende exclusivamente de la decisión autónoma y voluntaria.

Este marco descriptivo se relaciona directamente con el desarrollo de la conciencia moral social que forma parte de la ética del discurso, y que Apel y Habermas formularon a partir de los años setenta. Esta corriente ofrece un "fundamento de lo moral" que transforma dialógicamente el principio formal kantiano de la autonomía de la voluntad en el principio procedimental de la ética discursiva. Tal principio puede formularse del siguiente modo: "Todos los seres capaces de comunicación lingüística deben ser reconocidos como personas, puesto que en todas sus acciones y expresiones son interlocutores virtuales, y la justificación ilimitada del pensamiento no puede renunciar a ningún interlocutor y a ninguna de sus aportaciones virtuales a la discusión" (Cortina, 1996, p. 125).

La autora argumenta que Apel reconstruye desde una perspectiva dialógica dos conceptos clásicos en la ética occidental, es decir, los conceptos de persona y de igualdad. La persona se presenta como un interlocutor válido, que debe ser reconocido conforme pertenece a la comunidad de hablantes. A raíz de esto, la idea de igualdad se torna comunicativa, en la medida en que ningún interlocutor válido puede ser excluido de la argumentación cuando ésta versa sobre normas que le afectan o, en palabras de Habermas: "Sólo son válidas aquellas normas de acción con las que podrían estar de acuerdo todos los posibles afectados como participantes en un discurso práctico" (Cortina, 1996, p. 126).

En esta "teoría de la acción comunicativa" desarrollada por el pensamiento habermasiano, la conducta racional de las personas para elegir los medios más apropiados y para lograr un cierto fin, eventualmente puede lograrse mediante la acción comunicativa o comunicación lingüística. Se constata que el entorno inmediato de experiencias y vivencias, de certezas, evidencias y realidades, ese mundo de vida o trasfondo a partir del cual los sujetos pueden actuar de forma comunicativa produciendo y reproduciendo valores y normas destinadas al entendimiento mutuo y desarrollar interrelaciones de acuerdo con valores, está siendo trasplantado por un conjunto de fines instrumentales procedentes de lo que Habermas denomina sistema (Benabent, 2010, p. 130). 
El autor subraya que este sistema lo conforman las estructuras de orden económico y administrativo que se han desarrollado de forma autónoma presididas por la racionalidad instrumental, orientada a fines, y en las que el dinero o el poder se han convertido en los medios de integración. El planteamiento de Habermas se orienta a la posibilidad de revertir la tendencia y reconstituir la esfera pública, es decir, romper el dominio del objetivismo científico, redescubrir el mundo de vida y conformar una clase diferente de objetividad mediante la racionalidad comunicativa, que se hace posible a través del pensamiento libre y el discurso abierto, superando la racionalidad instrumental para reconstruir una racionalidad práctica y moral (Benabent, 2010, p. 130). De acuerdo al autor,

las condiciones para que todo agente que actúa comunicativamente participe en un proceso de entendimiento, tiene que elegir una expresión inteligible para que hablante y oyente puedan entenderse entre sí; el hablante tiene que tener la intención de comunicar un contenido proposicional verdadero para que el oyente pueda compartir el saber del hablante; el hablante tiene que querer expresar sus intenciones de forma veraz para que el oyente pueda creer en la manifestación del hablante (pueda fiarse de él); el hablante tiene, finalmente, que elegir una manifestación correcta por lo que hace a las normas y valores vigentes, para que el oyente pueda aceptar esa manifestación, de suerte que ambos, oyente y hablante, puedan concordar entre sí en esa manifestación en lo que hace a un transfondo normativo intersubjetivamente reconocido (Benabent, 2010, p. 131).

La meta del entendimiento es producir el acuerdo, de manera que los participantes en el proceso dialógico acepten un saber como válido, lo que para Habermas supone que es intersubjetivamente vinculante, es decir, que estas convicciones compartidas vinculan a los participantes en la interacción en términos de reciprocidad.

En el marco de esta perspectiva y por medio de la idea de una ética discursiva, "una norma únicamente puede aspirar a tener validez cuando todas las personas a las que afecta consiguen ponerse de acuerdo en cuanto participantes de un discurso práctico (o pueden ponerse de acuerdo) en que dicha norma es válida" (Habermas, 1991, p. 86). Habermas desecha, así, toda suerte de a priori metafísicos, de manera que las normas o deberes se encontraran, así, justificadas por medio de argumentos (Letelier, 2011). Esta idea de lo justo y lo bueno, y a su vez, vinculada al contexto jurídico, aleja la visión habermasiana de la orientación utilitarista, y se enmarca en 
lo que se conoce como "éticas dialógicas" desarrolladas en el siglo XX, dentro de las cuales es posible mencionar la "teoría de la justicia social" de John Rawls. En este sentido, el autor expresa "nuestro tema es de Justicia social. Para nosotros el objeto de la justicia es la estructura básica de la sociedad, más exactamente, el modo en que las instituciones sociales más importantes distribuyen los derechos y deberes fundamentales y determinan la división de las ventajas provenientes de la cooperación social. Por instituciones más importantes entiendo la Constitución Política y las principales disposiciones económicas y sociales" (Rawls, 1997, p. 20).

Algunos autores postulan que la teoría de la justicia trata primordialmente del problema de la filosofía política en los momentos actuales, es decir la fundamentación racional de las bases de la convivencia social y política, o citado en lenguaje filosófico y político, el problema de la legitimación del orden político. Esto es válido si se reafirma la búsqueda de una guía normativa mínima, es decir, racional, que posibilite un punto de referencia para el enjuiciamiento ético de las instituciones y la práctica política (Osorio, 2010).

Al comprender la sociedad como un sistema de cooperación cuyo propósito es velar por los intereses de sus miembros, se plantea una dificultad asociada a la organización de los acuerdos e intereses personales, los cuales están en permanente conflicto. Si bien se desprende que la sociedad no obedece a los criterios de justicia, sino que realza los intereses personales por sobre una concepción pública de justicia que permitiría la asignación de derechos y deberes, por un lado, y una distribución justa de los beneficios y las obligaciones de la vida cooperativa, por otro, el principal obstáculo radica en que los individuos no han sido capaces de llegar a acuerdos razonables de manera colectiva (Osorio, 2010, p. 141). Esta posición razonable para todos, que no es únicamente racional, sino también razonable, es en el enfoque del autor el punto central de la investigación rawlsiana y que se encuentra en su producción teórica bajo el nombre de "Posición Original".

Según el planteamiento de Rawls,

aquí el concepto intuitivo es que esta estructura (la sociedad) contiene varias posiciones sociales y los hombres nacidos en posiciones sociales diferentes tienen diferentes expectativas de vida, determinadas en parte, tanto por el sistema político como por las circunstancias económicas y sociales. De este modo las instituciones de una sociedad favorecen ciertas posiciones sociales frente a otras. Estas son desigualdades especialmente profundas $y$, sin embargo, no pueden ser justificadas apelando a las nociones de mérito o desmérito. Es a estas desigualdades de la 
estructura básica de la sociedad, probablemente inevitables, a las que deben aplicar en primera instancia los principios de la justicia social. Estos principios regulan, pues, la selección de una constitución política y los elementos principales del sistema económico y social (Rawls, 1997, p. 20).

En otras palabras, en términos rawlsianos se trata de refrendar la idea de una sociedad en que los seres humanos puedan vivir de manera libre e igualitaria, en un contexto de justicia igualitaria.

Hasta este punto se ha reflexionado acerca de la responsabilidad del individuo en la tierra, así como la explotación de los recursos, y la posibilidad única que posee para cambiar este escenario catastrófico, según consigna la ciencia apoyada en estudios y tecnocracia. Asimismo, se ha analizado a partir de diversas corrientes de pensamiento, la capacidad única de los humanos dentro del reino animal para generar acuerdos, al actuar de manera comunicativa y discursiva, posibilitando la generación de consensos colectivos, la conformación de una sociedad que actúe en el marco de la justicia social y, sobre todo, la organización de los sujetos en favor de sus demandas asociadas, no individuales ni codiciosas, y que propendan a la consolidación de un marco ético, político y participativo que vele por el cuidado del medioambiente y la calidad de vida de generaciones presentes y futuras.

En este sentido, Harvey (2001) sostiene que todas las políticas, sin importar su tipo ni el carácter de su objetivo (local, urbano, regional, nacional o planetario), tiene su origen en el desarrollo colectivo de una visión política determinada, por parte de personas determinadas, en lugares y momentos determinados. Si bien reconoce la existencia de una corriente de base presente en todos los lugares y localidades, sus intereses, objetivos y formas organizativas son generalmente fragmentarios, múltiples y de intensidad variada. El autor se pregunta el momento y la forma en que estos particularismos se vuelven coherentes internamente e integrados en una política más amplia.

Uno de los desafíos que el autor menciona es la relación dialéctica entre los movimientos de base y los procesos sociales más generales. El problema de la gran gama existente de luchas localizadas y particularistas es trascender la particularidad y alcanzar una concepción más global o universal de la política. Para los movimientos de oposición (opuestos a aquellos dedicados a reforzar la situación existente) esto significa definir una alternativa a ese sistema social que es la fuente de sus dificultades. Los movimientos de base sólo adquieren interés para el teórico o el partidario del cambio social en la medida en que trasciendan a dichas particularidades (Harvey, 2001, p. 210). 
Uno de estos movimientos de base, y que se entronca directamente con la materia discutida a lo largo de este acápite, corresponde al de justicia ambiental. Esta consideración ética dota de un sentido profundamente social y humanista a la discusión planteada, particularmente al considerar que la población mundial no se ve afectada de igual manera por las vicisitudes de origen natural o antrópico, sino que existen disparidades de acuerdo al estrato socioeconómico, la cultura, la etnia u otros factores que acrecientan la vulnerabilidad. Este movimiento surge en los Estados Unidos en la década de 1980, como resultado de un movimiento popular en contra de la instalación de un vertedero de policlorobifenilos en la provincia de Warren, Carolina del Norte, la cual era habitada en su mayoría por población de raza negra, quienes, al conocer la decisión gubernamental, optaron por las protestas de resistencia pacífica para que se respetase su derecho a la salud y a un medio ambiente sano, comenzando a acuñarse el término de justicia ambiental. Las protestas se caracterizaban porque sus integrantes eran de color y estaban apoyados por entidades religiosas, lo que impulsó el uso del término racismo ambiental, porque se consideraba denigrante la ubicación de ese tipo de depósitos en zonas habitadas por afroamericanos de bajos ingresos (Ramírez et al., 2015, p. 228). Los autores aseveran que, bajo la premisa del racismo ambiental, estos movimientos incitaron a otras comunidades a manifestar su malestar, reportándose protestas en Los Ángeles, Nueva York y desde Houston hasta Chicago, en las cuales ya no solamente participaban afrodescendientes, sino también población hispana. A partir de ese momento se comenzó a integrar el concepto de justicia ambiental, entendida como la reivindicación de derechos por actividades contaminantes, en virtud de la evidencia de riesgo ambiental y salud sobre las comunidades de bajos ingresos (Ramírez et al., 2015).

Espinosa (2012), por su parte, aclara que este contexto no debe entenderse como un rechazo a los principios de la corriente ecológica dominante ni como un enfrentamiento contra la población blanca, al contrario, el carácter eminentemente afroamericano del movimiento en sus inicios, y que posteriormente se abrió a otras etnias y amplió su ámbito de lucha a los sectores vulnerables del mundo, forma parte del imaginario racial que ha acompañado la lucha por los movimientos civiles en Norteamérica durante el siglo XX. Esto implica una ética de cuidado al medioambiente y también de los seres humanos independientemente de su condición social o pertenencia étnica.

A juicio de la autora, el instrumento teórico creado por el activista y profesor universitario Robert Bullard, está formado por una serie de principios 
y conceptos que buscaban invertir el paradigma de protección ambiental dominante en los Estados Unidos, el cual es aplicable en un contexto global. El marco de Bullard se conecta además con los otros dos grandes temas de la justicia ecológica; la inserción de los criterios de justicia ambiental en el desarrollo sostenible relaciona a este movimiento con la justicia intergeneracional y la invocación del principio preventivo lo ubica dentro de la teoría de la precaución. De la teoría planteada por Bullard se pueden extraer tres principios esenciales: i) todos los individuos tienen derecho a estar protegidos de la degradación medioambiental; ii) el principio de precaución, el cual establece que previo a emprender una acción, si se tiene una sospecha razonable de que puede producir algún daño y hay incertidumbre científica al respecto, entonces se debe actuar para impedir dicho daño, evaluando las alternativas para hallar la manera menos dañina de llevar a cabo dichas actividades, usando un proceso de toma de decisiones abierto, informado y democrático, que incluya a las personas que serán afectadas por la decisión y iii) la finalidad de invertir el paradigma de prevención dominante que, según los defensores de la justicia ambiental, refuerza el statu quo, la estratificación social y el impacto discriminatorio de la contaminación sobre las comunidades más vulnerables (Espinosa, 2012).

El movimiento se fue expandiendo y universalizando a partir de la década de 1990. Desde una perspectiva teórica, la justicia ambiental ha evolucionado a través de la apertura de su lenguaje y conceptos básicos. No obstante, más allá de la reflexión teórica, la expansión de la justicia ambiental en la actualidad se inserta en la lucha contra la globalización y sus efectos sobre las poblaciones más vulnerables. Al respecto, se considera que las empresas transnacionales tienen más facilidades para deslocalizar sus actividades hacia áreas con menos regulación medioambiental, más incentivos fiscales y grandes beneficios a un coste ínfimo y cuya expresión territorial se refleja en la extracción global de materias primas y minerales, localizados generalmente en áreas empobrecidas. Para los líderes de la justicia ambiental afroamericanos, en lo que a las transnacionales estadounidenses se refiere, sus actos no son más que la extrapolación de la política nacional basada en presionar a las comunidades más vulnerables y con menos recursos (Espinosa, 2012, p. 60).

El análisis presentado no tuvo como propósito generar una indagación absoluta acerca de los documentos y principios surgidos a partir de la "Ética de la Tierra" de Lepold, al contrario, el intento se vinculó más bien con la inclusión de variadas aristas que pudieran representar una mayor comprensión de la ética ambiental y su interrelación con la ciencia geográfica. 
$\mathrm{Al}$ respecto, la ética de la tierra fue el primer paso para realzar la responsabilidad del ser humano en la degradación del ambiente, y el necesario cambio de rumbo que, sin embargo, no se ha instaurado; al contrario, los intereses individuales, políticos y económicos han impedido este paso, y se han degradado ambientes, sin contemplar un marco mínimo de justicia social y ambiental, especialmente si el propio marco legal y acción gubernamental soslaya estas acepciones. En suma, se trata de abandonar la práctica de institucionalización de la vulneración a determinadas poblaciones, como se ha efectuado hasta hoy, y considerar que todos los sujetos además de poseer derechos y deberes, actúan en concordancia ética por cuanto despliegan su capacidad política de participación a través del intercambio comunicacional con otros individuos. Esto además constituye una doble responsabilidad a la humanidad en el cuidado del medioambiente, motivo suficiente para incorporar en la discusión dos conceptos gravitantes; el de justicia social y ambiental y el ordenamiento racional y equilibrado del territorio. Finalmente, y como señala Kant: "Obra de tal manera que trates a la humanidad tanto en tu persona como la de cualquier otro, siempre al mismo tiempo como un fin y nunca solamente como un medio" (Cortina, 2002).

\section{REFERENCIAS}

Arcas, P. (2007). Hans Jonas y el principio de responsabilidad: del optimismo científico técnico a la prudencia responsable (tesis doctoral). Universidad de Granada, España.

Attfield, R. (2005). "Generaciones futuras: Considerando todas las partes afectadas". Isegoría, 32, 35-46.

Benabent, M. (2010). "El interés general en la filosofía política. Un concepto ético y normativo necesario para la planificación territorial". Boletín de la Asociación de Geógrafos Españoles, 53, 121-143.

Carson, R. (1963). Silent Spring. Boston: Houghton Mifflin Company.

Cortina, A. (1996). "El estatuto de la ética aplicada. Hermenéutica crítica de las actividades Humanas". Isegoría, 13, 119-134.

. (2002). "La dimensión pública de las éticas aplicadas". Revista Iberoamericana de Educación, 29, 45-64.

. (2011). "Una propuesta de ética del desarrollo". Éthique et économique/ Ethics and Economics, 8(1), 25-39.

De Siqueira, J. (2001). "El principio de responsabilidad de Hans Jonas". Acta Bioethica, 7(2), 277-285.

Espinosa, A. (2012). "La justicia ambiental, hacia la igualdad en el disfrute del derecho a un medio ambiente sano". Universitas. Revista de Filosofía, Derecho y Politica, 16, 51-77. 
Ehrlich, P. (1968). The Population Bomb. New York: Ballantine Books.

González, G. (2015). "El principio de responsabilidad de Hans Jonas a la luz de la conciencia ecológica". Nómadas. Revista Crítica de Ciencias Sociales y Jurídicas, 46(2), 1-22.

Habermas, J. (1991). Conciencia moral y acción comunicativa. Barcelona, España: Editorial Península.

Harvey, D. (2001). Espacios del capital. Hacia una geografía crítica. Madrid, España: Editorial Akal.

Hervé, D. (2010). "Noción y elementos de la justicia ambiental: directrices para su aplicación en la planificación territorial y en la evaluación ambiental estratégica”. Revista de Derecho, 20(1), 9-36.

Jonas, H. (2001). Más cerca del perverso fin y otros diálogos y ensayos. Madrid, España: Editorial Los Libros de la Catarata.

Lecaros, J. (2013). La ética medio ambiental: principios y valores para una ciudadanía responsable en la sociedad global. Acta Bioethica, 19(2), 177-188.

Leopold, A. (2007). "La ética de la tierra". Revista Ambiente y Desarrollo, 23(1), 29-40. [Traducido de Aldo Leopold, A Sand County Almanac with Essays on Conservation from Round River, Ballantine, Nueva York, 1966.Traducción de Ricardo Rozzi y Francisca Massardo].

Letelier, R. (2011). "La Justicia Constitucional en el pensamiento de Jürgen Habermas”. Estudios Constitucionales, 9(2), 377-394.

Marcos, A. (2000). Ética ambiental. Valladolid, España: Universidad de Valladolid.

Meadows, D.H.; Meadows, D.L.; Randers, J.; Behrens, W.W. (1972). The Limits to Growth. New York: Universe Books.

Osorio, S. (2010). "John Rawls: una teoría de justicia social su pretensión de validez para una sociedad como la nuestra". Revista de Relaciones Internacionales, Estrategia y Seguridad, 5(1), 137-160.

Ramírez, S.; Galindo, M.; Contreras, C. (2015). “Justicia ambiental. Entre la utopía y la realidad social”. Revista Culturales, Universidad Autónoma de Baja California Mexicali, México, 3(1) 225-250.

Rawls, J. (1997) Teoría de la justicia. México: Fondo de Cultura Económica.

Rozzi, R. (2007). "De las ciencias ecológicas a la ética ambiental”. Revista Chilena de Historia Natural, 80, 521-534.

Sen, A. (1998). Las teorías del desarrollo a principios del siglo XXI. En: Emmerij, L. y Núñez, J. (Eds.), El desarrollo económico y social en los umbrales del siglo XXI (pp. 73-100). Banco Interamericano de Desarrollo, Washington, D.C.

Villarroel, R. (2007). “Ética y medioambiente. Ensayo de hermenéutica referida al entorno". Revista de Filosofía, 63, 55-72. 